\title{
Automatic Generation of Bi-Ventricular Models of Cardiac Electrophysiology for Patient Specific Personalization Using Non-Invasive Recordings
}

\author{
Karli Gillette ${ }^{1}$, Anton Prassl ${ }^{1}$, Jason Bayer ${ }^{2}$, Edward Vigmond ${ }^{2}$, Aurel Neic ${ }^{1}$, Gernot Plank ${ }^{1}$ \\ ${ }^{1}$ Institute of Biophysics, Medical University of Graz, Graz, Austria \\ ${ }^{2}$ LIRYC Electrophysiology and Heart Modeling Institute, Bordeaux Foundation, Pessac, France
}

\begin{abstract}
Introduction: Personalized in silico models of cardiac electrophysiology based on non-invasive recordings, such as body surface potential maps, are considered of pivotal importance in clinical modeling applications. Efficient, automated workflows are desired to construct patientspecific models for clinical use.

Objective: We aimed to develop an automated workflow for the generation of a parameterizable cardiac EP model capable of simulating body surface potential maps independent of user interaction.

Methods: A cardiac bi-ventricular model with torso was segmented and meshed from clinical MRI scans. Universal ventricular coordinates were computed for userindependent definition of fibers, a fast conducting endocardial layer, and earliest activation on the endocardium. The extracellular epicardial potential distribution was simulated and projected to the torso surface to acquire a body surface potential map.

Results: Total model generation from segmentation required approximately 2 hours. Automatized simulation of a single depolarization sequence required approximately 30 minutes using a forward element method implementation. Discussion: The proposed workflow integrated recentlydeveloped technologies to generate a parameterizable cardiac EP model within clinical time scales.
\end{abstract}

\section{Introduction}

Cardiac models of electrophysiology (EP) have been extensively used to better understand and characterize mechanisms of cardiac arrhythmic disorders. Due to advancements allowing for the efficient generation of anatomically specific models from clinically-based imaging data, as well as increased computational efficiency of cardiac tissue simulations, cardiac EP models have also become increasingly viable for clinical use in treatment planning and diagnostics. Recent applications including ablation guidance [1] and better clinical diagnostics measurements
[2].

Regardless, clinical integration is still limited by the personalization of such models for electrophysiological parameters based solely on non-invasive, commonplace clinical data such as the 12 lead electrocardiogram (ECG). Such personalization requires the development of efficient, automated workflows capable of generating cardiac models of EP within clinical time scales. Furthermore, resultant be models must compatible with machine learning, data assimilation, and optimization frameworks for personalization and therefore must be capable of user-independent parameterization.

To address this limitation, we aimed to generate an efficient, automatic workflow capable of generating adaptive cardiac EP models from clinical imaging data compatible with clinical time scales. In particular, an automated workflow was constructed to recover body surface potential maps (BSPMs), and thus 12 lead ECGs, simulated on an anatomically-specific bi-ventricular (BV) and torso model segmented from clinical MRIs. Mapped depolarization sequences on the $\mathrm{BV}$ were simulated according a confined electrophysiological parameter space required for patient-specific personalization. The workflow aimed to integrate various novel techniques for segmentation [3], model generation [4,5], and simulation [6] to allow for increased computational efficiency and automation.

\section{Methods}

The proposed workflow is shown in Figure 1.

A normal, healthy male patient underwent MR imaging directly following the acquisition of a clinical 12 lead ECG recorded using MRI-compatible electrodes. With electrodes intact, a full torso MRI at $2.5 \mathrm{~mm}$ resolution using T1 mapping was attained using four sequential acquisitions each taken at the same stage in the respiratory cycle. A higher-resolution $(1.5 \mathrm{~mm})$ iso-volumetric acquisition cardiac MRI was simultaneously attained.

Using semi-automatic approaches in Seg3D [7], a general torso and heart mask were segmented from the torso MRI. A four chamber heart was segmented from the car- 


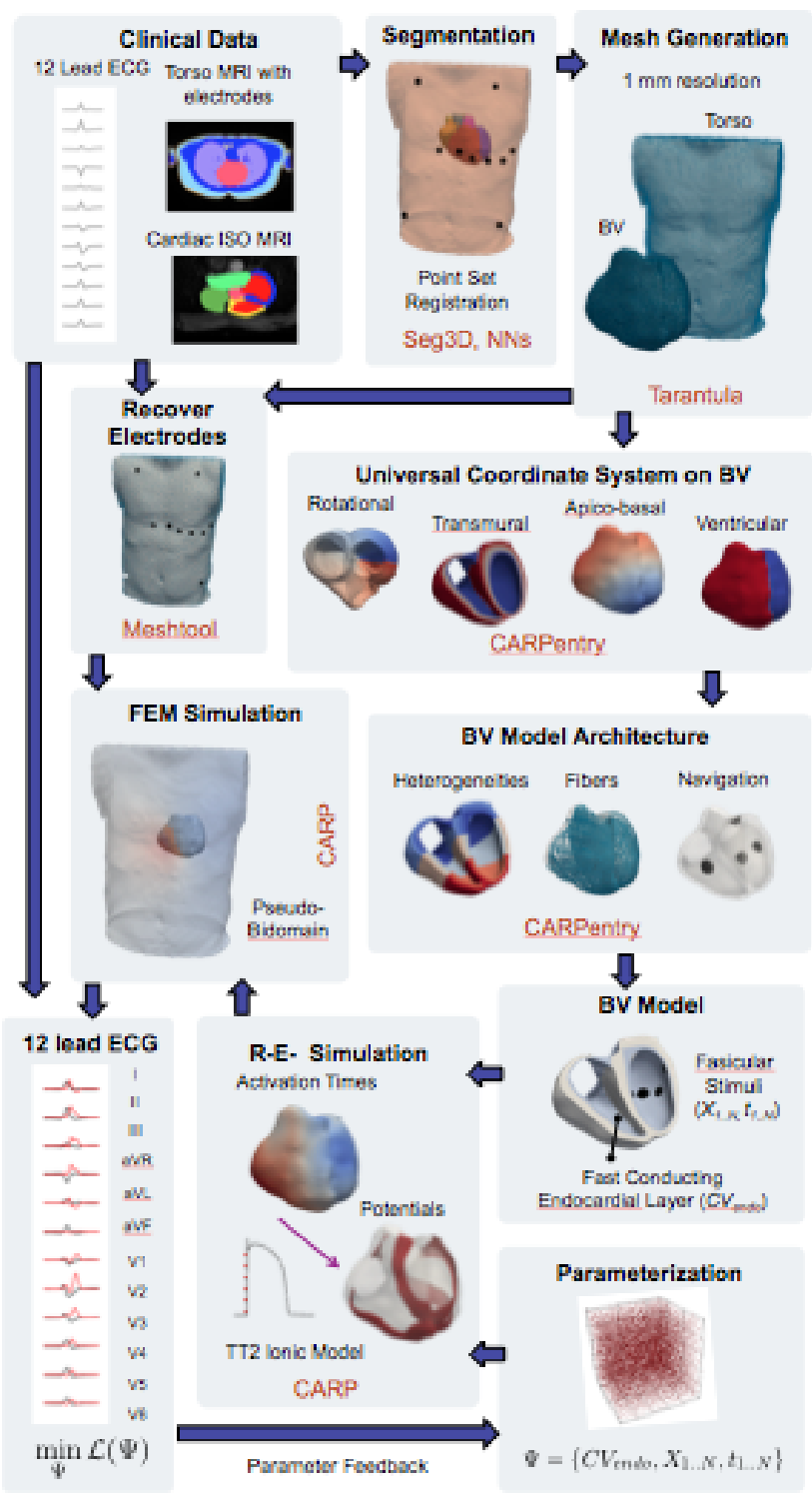

Figure 1: Workflow for semi-automatic generation of a cardiac EP model capable of simulating 12 lead ECGs.

diac MRI using a trained neural network [3] with manual post-correction. A conforming segmentation was generated by registering the $4 \mathrm{CH}$ into the torso using point set registration with the heart mask within Seg3D and meshed at $1 \mathrm{~mm}$ resolution using Tarantula. A BV was extracted for further mesh processing and subsequent simulation using meshtool.

A universal coordinate system (UVC) [5] implemented within the CARPentry Modeling Environment (https://carpentry.medunigraz.at/carputils/index.html) was constructed within the BV model allowing for navigation of stimulus sites, definition of heterogeneities, establish- ment of a rule-based-fiber architecture [4] assuming a rotational variation of -60 to 60 degrees through the ventricular free-wall. A fast-conducting endocardial layer substituted an explicit model for the His-Purkinje system. CV in the fast-conducting layer was assumed to be orthotropic with a prescribed principal velocity, $v_{\mathrm{ps}}$ and constrained ratios of $2 / 3$ and $1 / 3$ along the sheet and normal directions, respectively. Tissue conductivities were prescribed according to physiological values defined in literature [8].

Activation of the BV was initiated by three stimulus sites representing the septal $\mathbf{x}_{\mathrm{sf}}$, anterior, $\mathbf{x}_{\mathrm{af}}$, and posterior, $\mathbf{x}_{\mathrm{pf}}$, fascicles defined by UVC. For each site, timings, $\mathbf{t}$, and radii of direct activation, $\mathbf{r}$, were prescribed. The model could then parameterized with respect to input parameter vector $\mathbf{q}=$ $\left\{\mathbf{x}_{\mathrm{sf}}, \mathbf{x}_{\mathrm{af}}, \mathbf{x}_{\mathrm{pf}}, \mathbf{t}_{\mathrm{sf}}, \mathbf{t}_{\mathrm{af}}, \mathbf{t}_{\mathrm{pf}}, \mathbf{r}_{\mathrm{sf}}, \mathbf{r}_{\mathrm{af}}, \mathbf{r}_{\mathrm{pf}}, \mathbf{v}_{\mathrm{ps}}\right\}$.

A coupled reaction-Eikonal (R-E) model with diffusion [6] was used to sequentially compute an activation sequence and electrical source distribution throughout the BV model within CARP [9]. Briefly, the R-E model is equivalent to a standard reaction-diffusion monodomain model given as

$$
\beta C_{\mathrm{m}} \frac{\partial V_{\mathrm{m}}}{\partial t}=\nabla \cdot \boldsymbol{\sigma}_{\mathrm{i}} \nabla\left\{V_{\mathrm{m}}\right\}+I_{\text {foot }}\left(t_{\mathrm{a}}\right)-\beta I_{\text {ion }}
$$

$I_{\text {foot }}$ denotes an artificial current approximating an electrotonic current driving the foot of an action potential and triggered at activation times provided by the Eikonal equation [10]. A R-E model is advantageous due to the independency of computed source distributions upon spatial resolution enabling the use of coarser discretized meshes than normally feasible with plain reaction-diffusion models. The Eikonal equations were solved using an advancing wavefront approach [11]. Regional cellular dynamics in each region were represented by the tenTusscherNoble-Noble-Panfilov model [12] with region-specific adjustments [6].

A forward finite element FEM formulation using pseudo-bidomain implemented in CARP was used to compute a BSPM from the simulated BV potentials. A homogenous torso was defined as the volume conductor. Potentials at the recovered electrode positions correlating to nodes on the torso mesh were used to reconstruct QRS complexes of the clinical 12 lead ECG from the BSPM. Automated alignment with the reference 12 lead ECG [2] was performed and simulated leads were normalized to maximal and minimal amplitude across all leads allowing for quantitative comparison.

\section{Results}

Models constructed using the proposed workflow (Figure 1) are capable of automatically simulating QRS morphology of 12 lead ECGs according to a prescribed pa- 
rameter vector, q, within clinical time scales without user interaction. Timings for each component of the workflow is described within Table 1.

Table 1: Average computational times on a CPU with 10 cores for methodological tasks implemented within the workflow.

\begin{tabular}{cc} 
Method & Time $(\mathbf{m i n})$ \\
\hline Conforming Mesh Generation & 103.15 \\
Mesh Processing for FEM & 1.15 \\
BV Model Architecture & 17.28 \\
Reaction-Eikonal Simulation on BV model & 6.08 \\
Foward Mapping using FEM & 31.24 \\
\hline
\end{tabular}

Basic QRS morphology in the synthetic 12 lead ECG, constructed from the BSPM, was attainable (Figure 2) for a single set of parameters.
A

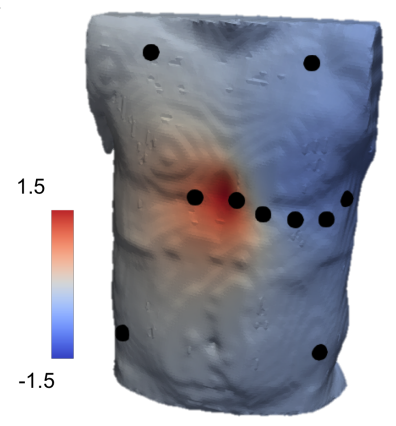

B

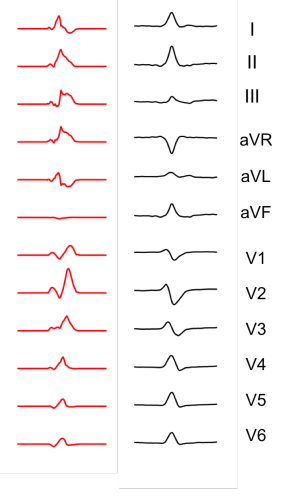

Figure 2: (A) BSPM map of the torso constructed using FEM formulation. Reference electrode placements used for 12 lead ECG reconstruction are shown. (B) Simulated QRS morphology of the 12 lead ECG (red) compared to a reference recording (black).

\section{Discussion}

The proposed workflow is a step toward further clinical integration of cardiac models of EP as it allows for generation of parametrizable and efficient models within clinical time scales based on clinically-attained imaging data. The resultant model was capable of automatically generating BSPMs functionalized with respect to a confined input parameter space $\mathbf{q}$ defining the stimulation and conductive profiles of the model and are therefore capable of patient-specific parameterization. Investigation into the influence of each parameter on QRS morphology, as well as validation of the generated QRS morphology, is currently underway. Patient-specific electrophysiological parameters of the model, which provide an optimal match between synthetic and reference ECG QRS morphology for sinus rhythm, will be attained using an extensive parameter sweep of the input parameter vector $\mathbf{q}$. It is proposed that a latin-hyper cube will be used for parameter sweep initialization and the loss metrics of L2 norm and cross correlation for defining quantitative match between simulated and reference 12 lead ECGs.

Various novel techniques for model generation and simulation have been utilized to construct a workflow within the CARPentry Modeling Environment that is capable of automatic generation of adaptive cardiac EP models within clinical time scales (Table 1). The novel techniques included a rule-based fiber architecture within the BV model and R-E with psuedo-bidomain for increased simulation speeds on coarser meshes. Additionally, automated navigation of stimulus points and a fast-conducting layer, and thus parameterizaiton of the model, was possible due to UVC. To increase computational efficiency of the workflow, thus allowing for more simulations in a shorter time, both a boundary element method formulation and coarsening of the torso mesh for finite element method (FEM) are under consideration for the forward projection of cardiac potentials. Furthermore, the role of conductive heterogeneity of the torso model will be explored through the addition of lungs. The replacement of the fast-conducting layer with a His-purkinje system using transfer mapping with UVCs is also being investigated.

\section{Acknowledgements}

This research was funded by BioTechMed, Austria as a part of the BioTechMed Flagship Project ILearnHeart. A special acknolwedgement to Dr. Ursula Reiter for data collection.

\section{References}

[1] Prakosa A, Arevalo HJ, Deng D, Boyle PM, Nikolov PP, Ashikaga H, Blauer JJ, Ghafoori E, Park CJ, Blake RC, et al. Personalized virtual-heart technology for guiding the ablation of infarct-related ventricular tachycardia. Nature Biomedical Engineering 2018;1.

[2] Arevalo HJ, Vadakkumpadan F, Guallar E, Jebb A, Malamas P, Wu KC, Trayanova NA. Arrhythmia risk stratification of patients after myocardial infarction using personalized heart models. Nature communications 2016;7:11437.

[3] Payer C, Štern D, Bischof H, Urschler M. Multi-label whole heart segmentation using cnns and anatomical label configurations. In International Workshop on Statistical Atlases and Computational Models of the Heart. Springer, 2017; 190-198.

[4] Bayer J, Blake R, Plank G, Trayanova N. A novel rulebased algorithm for assigning myocardial fiber orientation 
to computational heart models. Annuals of Biomedical Engineering 2012;40(10):2243-2254.

[5] Bayer J, Prassl AJ, Pashaei A, Gomez JF, Frontera A, Neic A, Plank G, Vigmond EJ. Universal ventricular coordinates: A generic framework for describing position within the heart and transferring data. Medical Image Analysis 2018;45:83-93.

[6] Neic A, Campos FO, Prassl AJ, Neiderer SA, Bishop MJ, Vigmond EJ, Plank G. Efficient computation of electrograms and ecgs in human whole heart simulations using a reaction-eikonal model, 2017.

[7] CIBC, 2016. Seg3D: Volumetric Image Segmentation and Visualization. Scientific Computing and Imaging http://www.seg3d.org.

[8] Roth BJ. Electrical conductivity values used with the bidomain model of cardiac tissue. IEEE Transactions on Biomedical Engineering 1997;44(4):326-328.

[9] Vigmond EJ, Hughes M, Plank G, Leon LJ. Computational tools for modeling electrical activity in cardiac tissue. Journal of Electrocardiology 2003;36:69-74.
[10] Colli Franzone P, Guerri L, Rovida S. Wavefront propagation in an activation model of the anisotropic cardiac tissue: asymptotic analysis and numerical simulations. Journal of mathematical biology 1990;28(2):121-176.

[11] Fu Z, Jeong WK, Pan Y, Kirby RM, Whitaker RT. A fast iterative method for solving the eikonal equation on triangulated surfaces. SIAM Journal on Scientific Computing 2011;33(5):2468-2488.

[12] Ten Tusscher K, Noble D, Noble P, Panfilov A. A model for human ventricular tissue. American Journal of Physiology Heart and Circulatory Physiology 2004;286(4):H1573H1589.

Address for correspondence:

Gernot Plank

Institute of Biophysics, Medical University of Graz, Harrachgasse 21, 8010, Graz, Austria

gernot.plank@medunigraz.at 